\title{
Tracking a Cellular Phone in a Stolen Phenomena
}

\author{
Mahfuzulhoq Chowdhury ${ }^{1}$, Talemul Islam ${ }^{1}$ and Parijat Prashun Purohit ${ }^{1}$ \\ ${ }^{1}$ Department of Computer Science \& Engineering, Chittagong University of \\ Engineering \& Technology, Chittagong, Bangladesh \\ mahfuz_csecuet@yahoo.com,talemul@yahoo.com,parijat2009@gmail.com
}

\begin{abstract}
Cellular phone is a method for communication. As innovation is quickly redesigning, it gives variety of work extension. Additionally it is likewise considered now days, stockpiling of huge information. In spite of the fact that loss of a phone because of robbery is a sudden event, it can be recouped by method for our executed android application. It gives location upgrade in at regular intervals through email. In the event that the mobile is turn on or restart then the application will run. A password will empower to turn off the application. This application is tested frequently in arbitrary cases and the precision level is attractive.
\end{abstract}

Keywords: Android, Location Tracking, Google Map, Location Based Services (LBS)

\section{Introduction}

Cellular phone technology is the most inclining innovation in today's reality. There are some new discharges in regards to cellular telephones. In addition it has turned into a part of individuals everyday life as an as often as possible utilization gadget. Mobile computing is human-computer cooperation by which a machine is required to be transported amid typical utilization. Versatile registering includes portable correspondence, versatile fittings, and portable programming. Correspondence issues incorporate impromptu and framework arranges and in addition correspondence properties, conventions, information arrangements and cement advances [1]. It additionally upholds the client with location based administrations and greetings tech frill gadget underpins on single gadget. Today very nearly every cellular phone has the peculiarities of GPS help, Internet, Speech to Text change and so on. Fast processor with overhauling working framework is likewise an extensive progression of becoming rate of high mobile phone software. A smart phone is a mobile phone which has more praiseworthy registering ability than a common cell telephone. It is based on a portable working framework. In certifiable the quantity of smart phone user is expanding for the gift of modern science and technology and the uses of smart phones spreads the most extreme vital work in every part of our life. The gadget size is little yet it is valuable in numerous angles. So the passing of a cell phone is a tiresome experience. Because of this event victimized people experience the ill effects of a great deal of issues for recuperation. Vital information may be lost which has no reinforcement. Along these lines, recuperation of lost cell phone is important in that sort of situation. Our application gives location interim of one minute through email. On the off chance that the SIM card is changed or the mobile is turn on or restart then the application will run. On the off chance that the client needs to stop the administration he/she can't fruitful without password. In the event that the password will match then the administration will stop. 


\section{Background and Related Works}

"Traintdroid" is proposed in [2] for monitoring third party applications. It is concerned to distinguish acting up applications in execution environment and it can be a method for security for delicate information of cell phone. The efforts to establish safety for current advanced cell is broke down [3]. An against robbery arrangement focused around locking SIM card of phone is proposed. The arrangement comprises of programming as per which fittings parts need to be chosen. By the utilization of programming calculation, clients may discover stolen cell telephone and ensure discriminating data. To some degree it can lessen the misfortunes brought about by the stolen telephone. Application [4] gives location when the SIM card is changed. Be that as it may if the SIM card is not changed, there is no chance to get of discovering area redesign. An alternate versatile application named "Where's My Droid" [5]. At the point when the cell phone sets out for some missing, it sends the GPS directions of the telephone. Then again, the client can remotely control one's telephone by associating it to the "Commandant", an electronic interface. It additionally offers a Pro form which gives you a chance to remote bolt the telephone rendering it impervious or remote wipe the application to spare your information from abuse. For security of cell phone and contact synchronization Gupta et al. proposed their framework [6]. It centered an online database where contact data of clients are put away. At the point when a client's cell phone or SIM card is changed, each contact who is synchronized to this database will be advised about client's upgraded data. Also it likewise proposes an antitheft arrangement. The application will store one or more predefined cell numbers. In the event that the SIM card is changed then the area overhaul will be told in those predefined cell numbers. Accordingly present area of the stolen phone can be followed. For this, a client will need to keep two mobile phones to track or rely on upon other cells for following the lost one. Area recognition technique is executed by approaching the target telephone [7]. They created two applications specifically "Microaddress Recorder" which will introduce on Mobile and "GPS area taking care of framework" which will run on program. Both applications will work at the same time for recording, putting away and showing the areas with simple interface to client. It expects to give a general outline of area following by ringing on focused on Mobile client furthermore a client interface to the web application so that there is no abuse by third individual. Vehicle antitheft following framework is proposed by Liu et al. [8]. A prescient following of versatile occasions utilizing cell telephone is proposed as a part of [9]. Here it is conceivable to track portable focuses through neighborhood sensing gadgets and future location of a target can be resolved utilizing a conveyed Kalman-Consensus sifting calculation.

Antitheft arrangements are proposed in mixed bag of works. All these arrangements can be connected however there are doubtlessly a few confinements. In addition some proposed framework relies on upon different elements e.g. SIM card change, option cell numbers and so on. In the event that these elements are not changed then the whole framework may neglect to track the area and accordingly there is no possible arrangement of these phenomena. Area redesign through email can be simpler arrangement and if change of SIM card is not required then precision of the arrangement will be better.

This paper is organized in several sections. Sections two contains some related works on location tracking as well as security measures, present state of the problem, motivation of the research and objectives. Section three contains brief discussion on Literature Review. In section four entire proposed systems is described with technical details. Evaluation of the proposed system and summary of overall system are discussed later. 


\section{Location Based Services}

\subsection{Fundamental of Location Based Services Application}

Location based services are a general class of computer program based administrations used to incorporate particular controls for location and time information as control peculiarities in computer programs. As being what is indicated (LBS) is a data and has various uses in Social Networking today as a stimulation administration, which is available with mobile phones through the portable system and which utilizes data on the land position of the mobile phone. This has gotten to be more paramount with the extension of the advanced cell phone and tablet showcases also. LBS are utilized as a part of an assortment of connections, for example, well being, indoor item seek, excitement, work, individual life, and so forth. LBS incorporate administrations to distinguish an area of an individual or item, for example, finding the closest managing an account money machine or the whereabouts of a companion or worker. LBS incorporate package following and vehicle following administrations. LBS can incorporate portable trade when taking the type of coupons or publicizing guided at clients focused around. The achievement and becoming rate of LBS engineering have propelled us to create another framework for Vaccination update framework. LBS can help us to identify one's position, follow his development, figuring out closest immunization focus. We utilize LBS with both the GPS and Network engineering at whatever point which one is accessible and productive to us. The Location Based Services (LBS) are versatile applications that rely on upon the location of the cell phone. LBS administrations can be ordered as forced LBS administrations (push administrations) and as client asked for LBS administrations (pull services).in request to make the LBS administrations conceivable, some foundation components are fundamental, including [10]:

- mobile devices,

- applications,

- communication network,

- positioning of components,

- $\quad$ servers,

- $\quad$ services.

Mobile devices are tools used to get to LBS administrations, send demands and right comes about. Such gadgets can be Personal Navigation Devices (Pnds), Portable machines, Mobile telephones, and so forth. The client's interface for access to LBS administrations speaks to an application. Normally it is programming created by the administration supplier, transferred and introduced on the client's gadget. The particular application is normally created for particular .LBS administrations. As a result of the stipulations of the cell phones (little show size, processor of constrained force and memory, battery limit), LBS applications must be light and spare batteries. Correspondence system alludes to portable systems that exchange the asked for administrations from the client to the administration supplier, and the asked for data again to the client. The Global System for Mobile Communications (GSM) is right now the most widely recognized standard for portable systems and utilized generally for cell telephones at the worldwide level. Portable systems are normally controlled and kept up by the administrators who give associations with the versatile clients and are in charge of the information and voice exchange.

The situating of parts is generally essential in LBS applications keeping in mind the end goal to focus the area of the client's cell phone. In the greater part of current LBS benefits the client is not needed to enter the area physically, nor the data of postcodes or road names. Rather, the position of the client gadget can be gotten by utilizing the situating engineering, for example, satellite situating, situating by portable system, WLAN stations or radio 
associations. The administration suppliers are occupied with server support which sends distinctive sorts of LBS administrations to clients, and are in charge of the preparing of administration appeals and for furnishing a proportional payback result. The server computes the positions, searches for the courses or particular data focused around the client's area. The administration suppliers normally keep up all data asked for by the client. Rather, the substance suppliers are in charge of the gathering and stockpiling of geographic information, area based data, and so on. These information will be asked for and handled by the server and after that came back to the clients.

\subsection{Principle of GPS}

GPS satellites round the earth twice a day in an extremely exact circle and transmit signal data to earth. GPS beneficiaries take this data and use triangulation to ascertain the client's careful area. Basically, the GPS recipient analyzes the time a sign was transmitted by a satellite with the time it was gotten. The time distinction tells the GPS collector how far away the satellite is. Presently, with separation estimations from a couple of more satellites, the collector can focus the client's position and presentation it on the unit's electronic guide. Taking the case of getting the position of a gadget with GPS signal recipient, the guideline of GPS is depicted as takes after [11]:

- First, the receiver measures the travel time of a pseudo-arbitrary code sent from a GPS satellite to the recipient. From the measured time, the receiver can compute the distance from the receiver to the satellite (x). So the beneficiary must be some place on the surface of a circle fixated on the satellite with range $x$ (Figure 1(a)).

- Then, the distance of the receiver to a second satellite is measured in the same way. Presently the potential area of the beneficiary is contracted to a circular ring at the convergence of the two circles (Figure 1(b)). The potential area of the recipient is contracted to two focuses by alluding estimations from a third satellite (Figure 1(c)).

- One of these positions can be denied because of some reasons, such as it is too far away from the Earth, or moves at impossible speed. By taking measurements from a fourth satellite, the receiver can be positioned to a point. The diagrams in Figure 1 show a simplified 2-dimensional model.

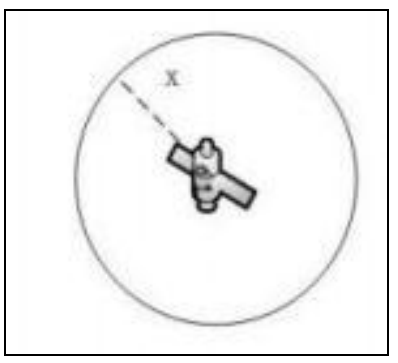

(a)

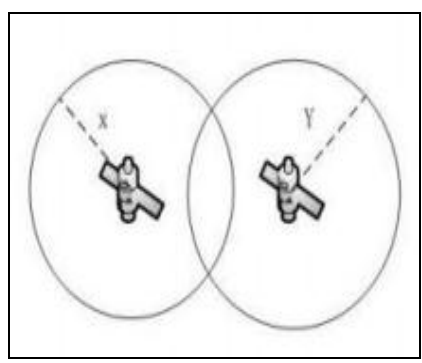

(b)

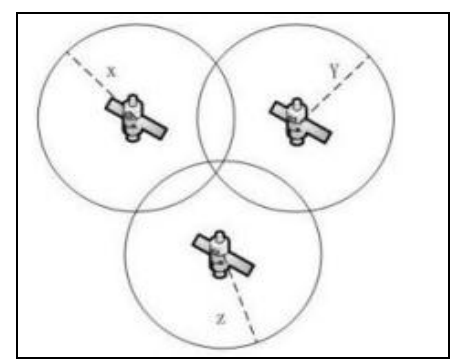

(c)

Figure 1. GPS Position Computation

\subsection{Positioning with GPS}

Meaning to determine our position on the earth is an usual method is to refer to a terrestrial position (i.e., position on the Earth) by its latitude and longitude. Therefore, most GPS receivers display their current latitude and longitude. The usual format for displaying this information is in degrees and minutes. There are 360 degrees in a complete circle, and 60 minutes in one degree. The familiar symbol for" degree" is ${ }^{\circ}$. The symbol for minute is ". The 
minutes are usually displayed as a decimal number, like 36.2536'. Both latitude and longitude are angles, and they therefore have to be measured in reference to a well-defined $0^{\circ}$ line. The most common way to locate points on the surface of the Earth is by standard, geographic coordinates called latitude and longitude. These coordinates' values are measured in degrees, and represent angular distances calculated from the center of the Earth. Figure 2 shows the angular distance of latitude and longitude.

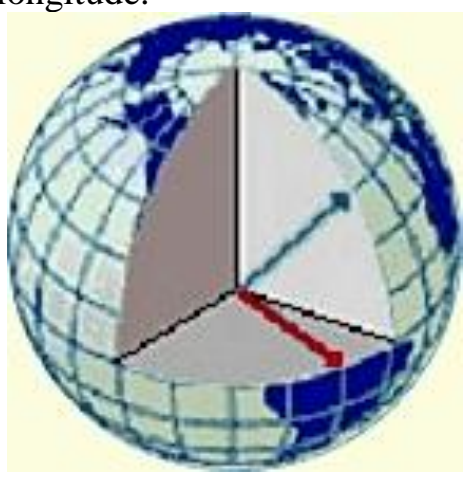

Figure 2. Angular Distances of Latitude and Longitude

\subsection{Choice of Operating System}

The Mobile Operating System (MOS) is a framework which deals with the cell phone, like a working framework (Linux or Windows) which controls a home machine. Presently they are to a degree client friendlier and arrangement more with remote techniques for wideband association and portable multi-media conceivable outcomes. The right now heading stages available are Android, Iphone, Symbian, Blackberry, Windows Phone and so on. Concerning transforms it is hard to focus decisively what is the offer of individual working framework available, yet the patterns, focal points and disadvantages of one stage in connection to an alternate or others are known. The clients yearnings and needs focus most the use of a certain stage yet sudden improvements and changes show that there is a fight available, and consequently likewise enormous headways making the client the greatest champ. In figure 3 we can see the detail of different portable working framework including android.

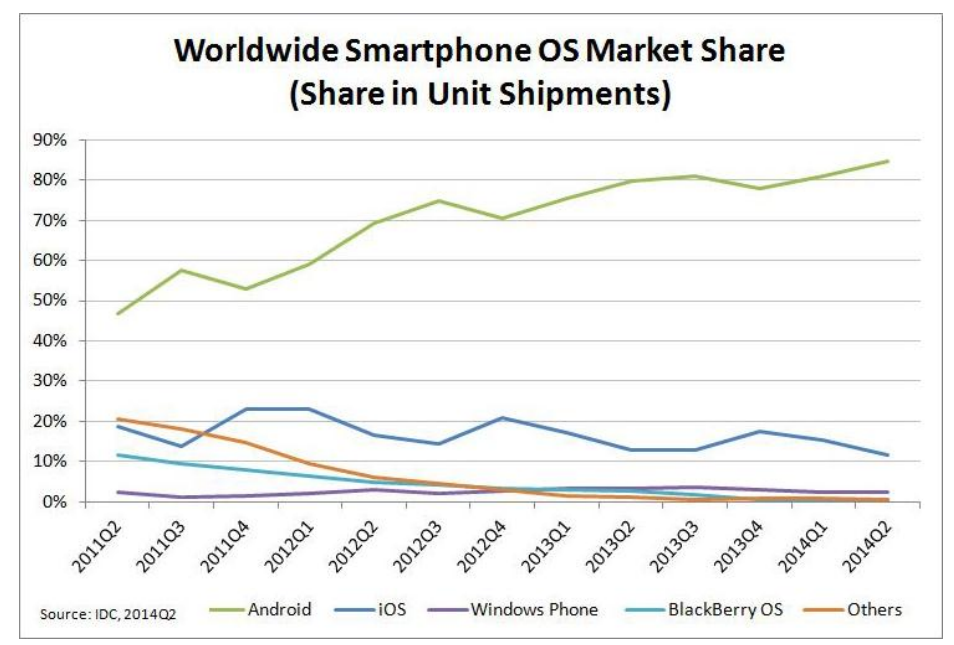

Figure 3. Statics of the Future Usage of Mobile Device Operating Systems 
Android is a software platform and operating system for mobile devices, in light of the Linux piece, and created by Google and later the Open Handset Alliance. It is a complete programming stack that gives all the middle ware required to run end-client applications on cell phones, for example, gadget drivers, OS, center libraries, an advanced virtual machine, Java Native Interface (JNI), and a complete Java advancement environment. Android is open source, significance engineers can change and tweak the OS for each one telephone. Consequently, distinctive Android-based telephones may have diverse graphical client interfaces Guis despite the fact that they utilize the same OS. Android telephones regularly accompany a few inherent applications furthermore help outsider programs. Engineers can make programs for Android utilizing the free Android SDK (Software Developer Kit).

\section{Technical Details of Proposed System}

\subsection{Preliminaries}

Location based Services offer numerous focal points to the portable clients to recover the data about their current location and process that information to get more helpful data close to their area. With the assistance of A-GPS in telephones and through Web Services utilizing GPRS, Location based Services can be actualized on Android based advanced mobile phones to give these quality included administrations: informing customers concerning current activity conditions, giving directing data, helping them find adjacent fir stations. In this paper, we propose the implementation of Location based services through Google Web Services and Walk Score Transit APIs on Android Phones to give multiple services to the user based on their Location. A basic view of location based service is in Figure 4.

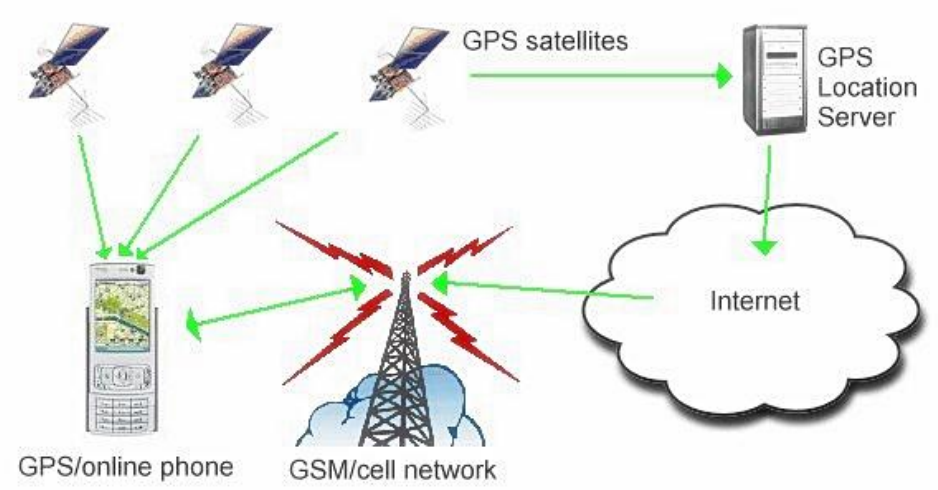

Figure 4. Location based Services

\subsection{Haversine Formula}

The haversine formula is a mathematical statement essential in navigation, giving extraordinary circle distances between two focuses on a sphere from their longitudes and scopes [12]. It is an unique instance of a more general equation in round trigonometry, the law of haversine, relating the sides and plot of circular triangles. We have utilized Haversine recipe [13] for ascertaining separation between two areas.

The formula is described as:

$\mathrm{R}=$ earth's radius (mean radius $=6,371 \mathrm{~km}$ )

$\Delta$ lat $=$ lat2 - lat 1

$\Delta$ long $=$ long2- long1 


$$
\begin{aligned}
& a=\sin ^{2}(\Delta \operatorname{lat} / 2)+\cos (\text { lat } 1) \cdot \cos (\text { lat } 2) \cdot \sin ^{2}(\Delta \text { long } / 2) \\
& c=2 \cdot \operatorname{atan} 2(\sqrt{ } a, \sqrt{ }(1-a)) \\
& d=\text { R.c }
\end{aligned}
$$

The above equations mathematically and computationally exact results for calculating the distance. The intermediate $\Delta$ lat calculates the difference in latitude of two points and $\Delta$ long also calculates the difference in longitude of two points. The intermediate result a is the square of half of the straight-line distance (chord length) between the two points. The intermediate result $\mathrm{c}$ is the great circle distance in radians. The great circle distance $d$ will be in the same units as $\mathrm{R}$ which is the distance between two places considering as two points e.g. (long1, lat1) and (long2, lat2).

\subsection{Proposed System}

When a mobile is lost or stolen by a thief then the thief must switched off and far away from that place. Then he switch on a safe place to sell or use the phone. But when he is switched on the mobile then the application will run and it active the GPS and mobile network. This application send location to the user mail in interval of one minutes. The user go to the location and catch the lost phone. Then the user stop the application by using his password. Figure 5 demonstrates the flowchart our application. The service continuously collect information the location manager of GPS provider. If GPS data is not available then service will not send any location. If the service get the GPS data constantly then send location through email after one minute interval. To calculate distance we use Harversine formula. This formula gives minimum difference between two points on a sphere from their latitude and longitude. The service will compare the difference with previous location. If the difference is less than one meter, then there will not send any location. Here the application call a thread after one minute to update the location. If the location is change the send location through email.

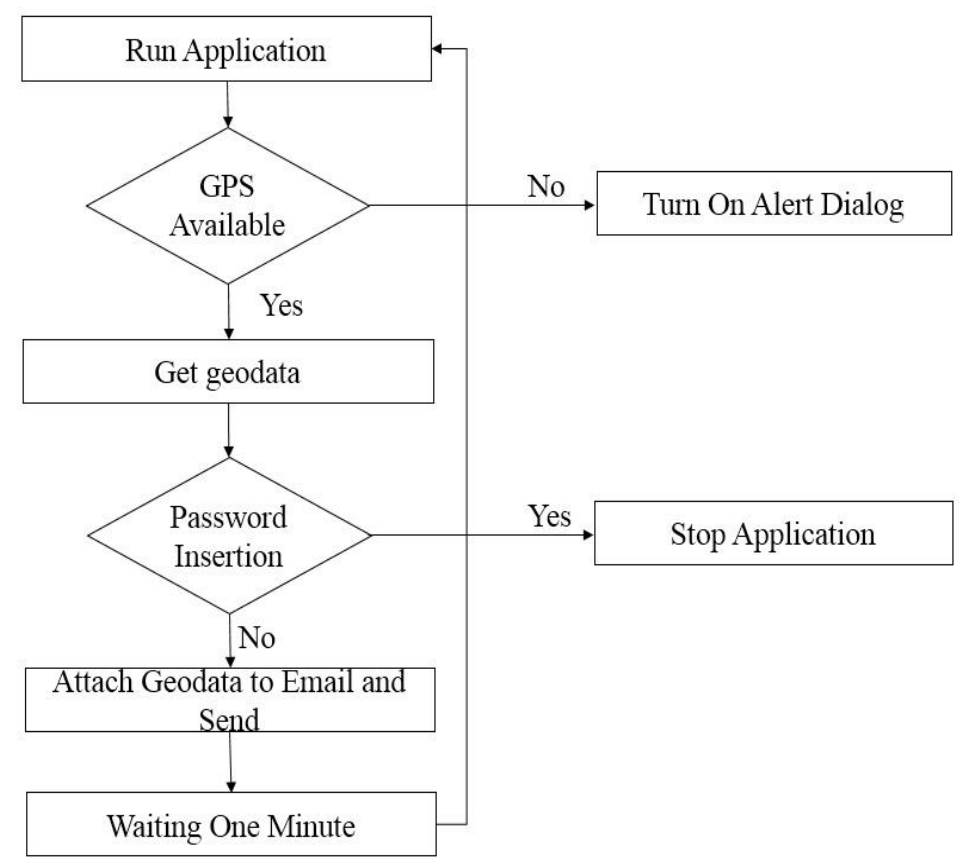

Figure 5. Flowchart of Proposed System Architecture 


\section{Performance Evaluation}

The sample mail that is received along with geo data is shown in Figure 6. Our main purpose is to track the stolen phone location.

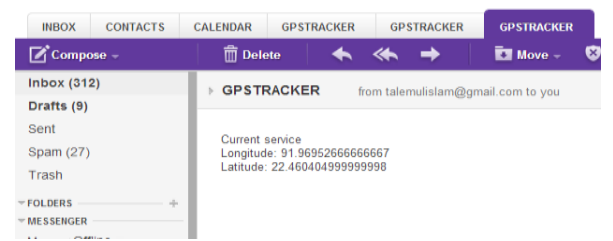

\section{Figure 6. Sample Email which is Auto Send by This Application}

Sequentially the updated positions are shown in Figure 7, 8 and 9 respectively. Here each time a new marker is added that indicates the change of location.

Latitude and Longitude of a Point

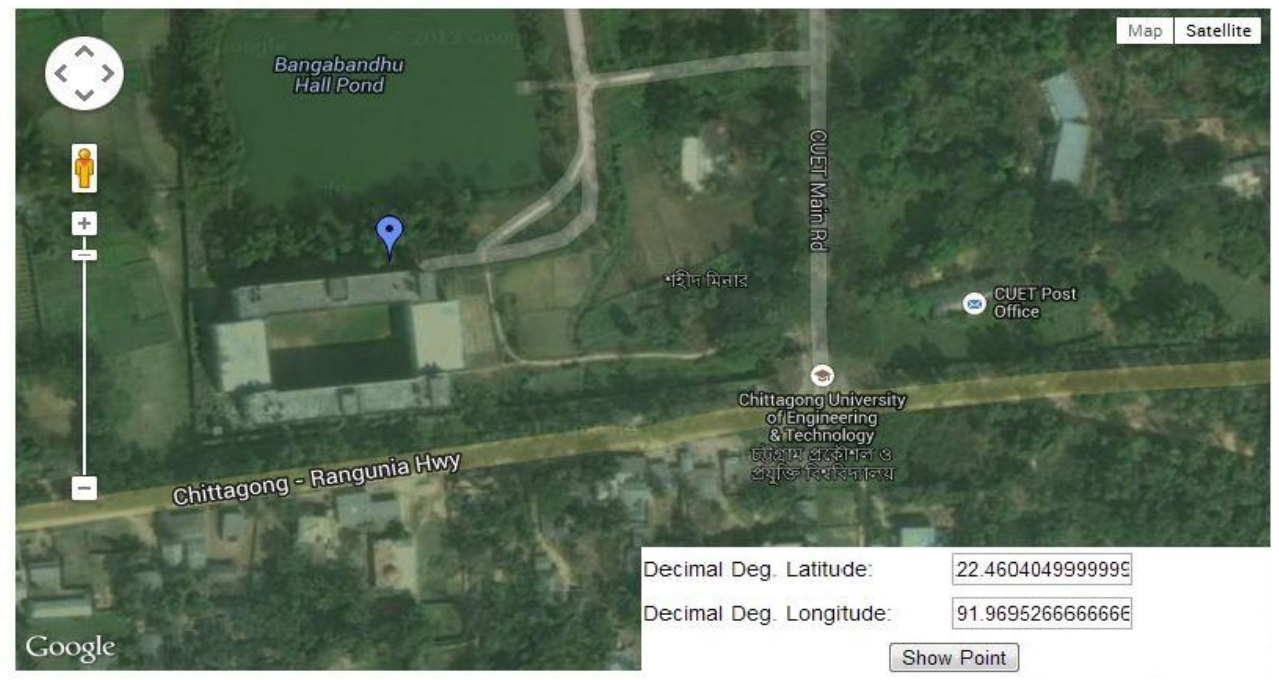

Figure 7. First Position

\section{Latitude and Longitude of a Point}

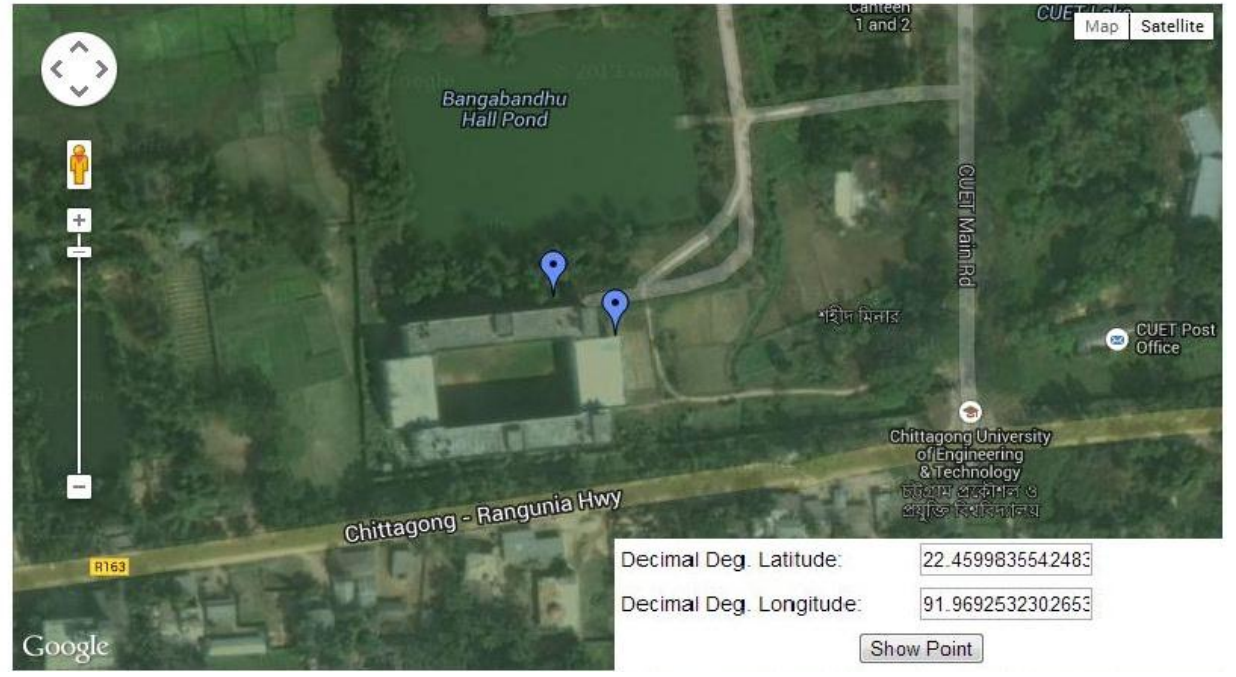

Figure 8. Second Position 


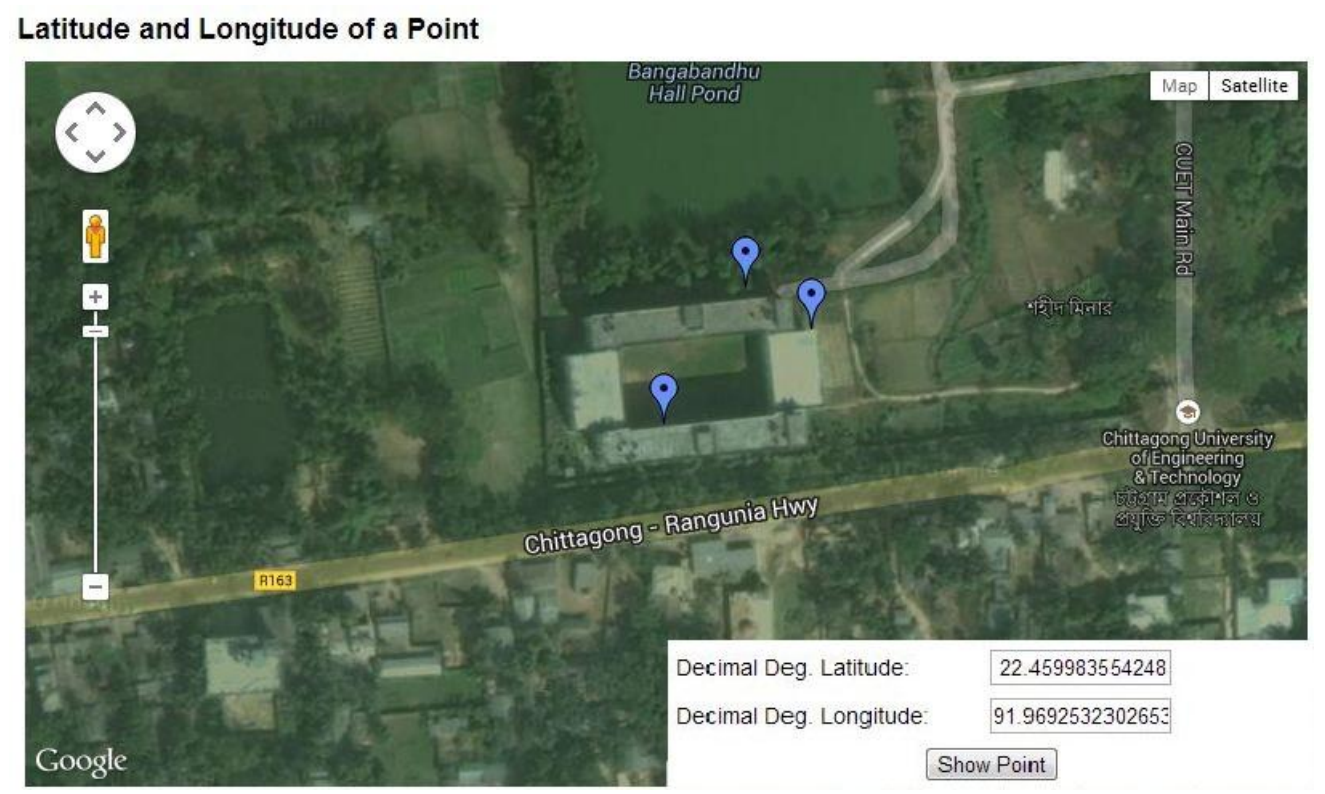

Figure 9. Third Position

We interviewed 100 cell phone users with three questions:

1. Do we need a mobile application for tracking stolen cell phone?

2. Do you think it will be helpful?

3. Will you use this app?

Figure-10 shows the responses in bar chart.

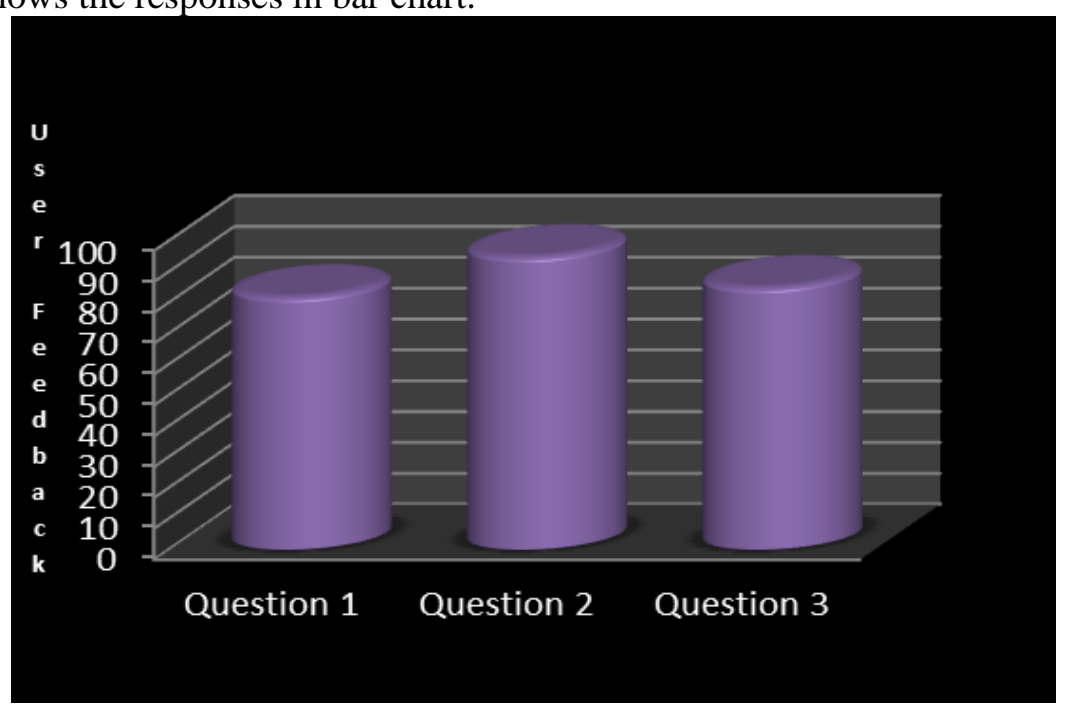

Figure 10. Response to Three Questions

We showed 100 people our developed application. They were told to choose from three options whether this was-

1. Very Helpful

2. Useful 
3. Need Improvement

Figure-11 shows it graphically. We will further improve our application based on their suggestions.

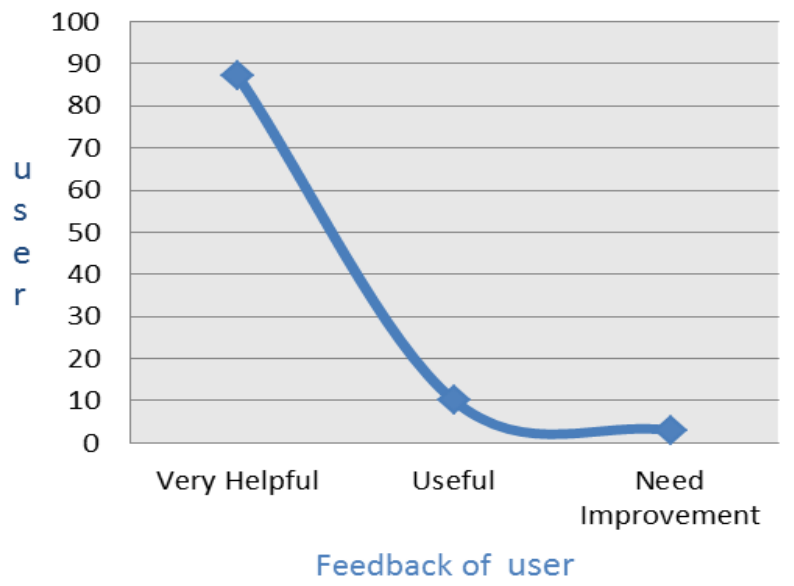

Figure 11. Response to Three Questions

\section{Conclusion and Future Recommendations}

Our application is actualized for the android based cellular phone. Comparative application can be produced for other operating systems. There are some limitations that can be future scope. If someone flash the mobile, then the application can't update location information anymore. But if the user lock the setting then no one is able to factory reset the phone. Moreover android developers can add this application as a built in application and lock the boot menu to flash so that it is not possible to steal the cellular phones.

\section{References}

[1] Mobile Computing, "http://en.wikipedia.org/wiki/Mobile_computing".

[2] William Enck, "TaintDroid: an information flow tracking system for real-time privacy monitoring on smart phones," Commun. ACM, vol. 57, no. 3, (2014), pp. 99-106.

[3] Gao, "A Smart Phone Anti-theft Solution Based on Locking Card of Mobile Phone", in proc. of international conference of Computational and Information Sciences (ICCIS), (2011), pp. 971 - 974.

[4] Last day of access (March 5,2013). [Online] Available:http://www.phonearena.com/news/SamsungDivehelps-getting-your-lost-or-stolen-smartphone-back_id20604.

[5] Last day of access (September 3,2013). [Online] Available: http://www.hongkiat.com/blog/track-lost-stolenandroid-devices/

[6] Gupta, "Smartphone Security and Contact Synchronization," in proc. of fourth International Conference on Communication Systems and Network Technologies (CSNT), (2014), pp. 621-625.

[7] S. Bhatia and S. Hilal, "Semantic description of mobile application for location tracking," International Conference on Optimization, Reliability, and Information Technology (ICROIT), (2014), pp. 267-272.

[8] Z. Liu, A. Zhang and S. Li, "Vehicle anti-theft tracking system based on Internet of things", in proc. of IEEE International Conference on Vehicular Electronics and Safety (ICVES), (2013) July, pp. 48-52.

[9] Perakovic, "Reminder based on the users's location", in proc. of ICTS 2011-Maritime, Transport and Logistics Science, pp.1-9,(2011).

[10] T. Chang, "Analysis of Critical Success Factors of Mobile Location-based Services", Master's thesis, Available from: tslab.ssvl.kth.se, Royal institute of technology, (2009) June 20.

[11] "Haversine Formula", http://en.wikipedia.org/wiki/Haversine_formula

[12] "Deriving Haversine Formula", http://mathforum.org/library/drmath/view/51879.html 

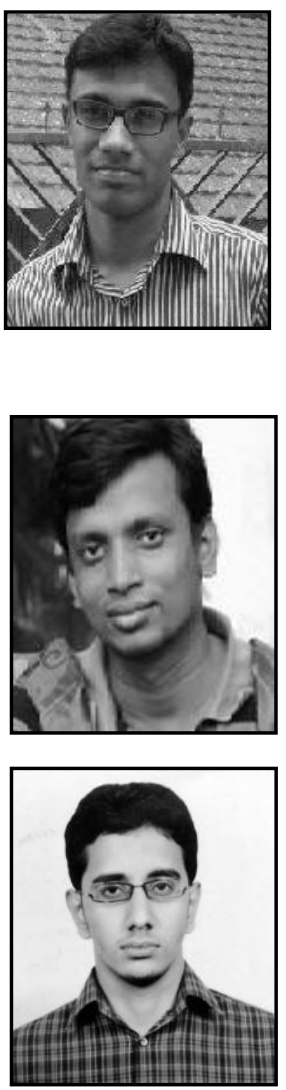

Mahfuzulhoq Chowdhury, he received the B. Sc. Engineering Degree in computer science and engineering from Chittagong University of Engineering and Technology, Bangladesh, in 2010. From September 2010 onwards he has been serving as a faculty member in the Department of Computer Science and Eng., Chittagong University of Engineering and Technology (CUET), Chittagong, Bangladesh. His major researches include Human Computer interaction, Cognitive Radio Networks, Cryptography, Wireless Sensor Networks etc.

Talemul Islam, he received the B. Sc. Engineering Degree in computer science and engineering from Chittagong University of Engineering and Technology, Bangladesh, in 2013. His major researches include Human Computer interaction, Cryptography, Android application development etc.

Parijat Prashun Purohit, he has completed his Bachelor of Science from Chittagong University of Engineering and Technology in the year 2014. His major was Computer Science and Engineering. His research interest includes Artificial Intelligence, Natural Language Processing and Cloud Computing. 
International Journal of $u-$ and e-Service, Science and Technology Vol. 7, No. 6 (2014) 